\title{
An Error Monitoring Algorithm for ATM Signalling Links
}

\author{
K. Kant and J. R. Dobbins \\ Bell Communications Research \\ 331 Newman Springs Road, Red Bank, NJ 07746, USA \\ Tel: (908)758-5384, Email: kant@perf.bellcore.com
}

\begin{abstract}
This paper proposes an error monitoring algorithm for in-service signalling links at the network node interface (NNI) using the ATM adaptation layer for signalling (SAAL). The algorithm is intended for use on links using the SSCOP (Service Specific Connection Oriented Protocol) of ATM adaptation layer 5. It uses only the information that is either already available from SSCOP or can be obtained easily. It does a better job in monitoring the link quality over a wide range of ATM network parameters (e.g., a wide range of link speeds, message length distributions, and offered loads) than known error monitoring algorithms with only one set of parameter values.
\end{abstract}

\section{Keywords}

ATM Adaptation Layer, Signalling, SSCOP, Error Monitoring, transmit congestion.

\section{INTRODUCTION}

The provision of switched virtual circuits (SVCs) in an asynchronous transfer mode (ATM) network requires a reliable and efficient signalling protocol. Such a protocol has already been standardized and is known as SSCOP, or service-specific, connection oriented protocol (Quinn 1993). SSCOP is a link level protocol and provides selective retransmission of lost messages (or protocol data units (PDUs)). A "link" in the ATM context is really a virtual circuit designated to carry signalling traffic. The bit rate for this virtual circuit could be anywhere from $64 \mathrm{~Kb} / \mathrm{sec}$ to $4 \mathrm{Mb} / \mathrm{sec}$ depending on the signalling needs. Since the availability of SSCOP links (or virtual circuits) is vital for all services using SVCs, it is important to perform error monitoring on these links. Although the lower layers (e.g., ATM and SONET) do provide a certain degree of error monitoring, an application-level error monitoring is also necessary to provide a tighter control over link quality. The error monitor presented here is a part of the algorithm accepted by ITU as an example of desirable SAAL error monitoring algorithms. 
A good design of an error monitor involves several considerations as discussed in (Ramaswami 1993, Kant 1994). This section motivates some of the major considerations.

In telecommunication networks, most network components, including links, are operated in the duplex mode, i.e., normally, the carried load is shared by two identical links. If one of these links is taken out of service, a changeover is initiated which transfers all undelivered messages to its "mate" link. When a repaired link is put back into service, a changeback process is initiated, which redistributes the traffic over the pair. These mechanisms provide immunity against single failures; however, they do involve a lot of overhead and message delays. Thus, if the link is hit with only a short error burst, it is better to keep the link in service even if the bit error ratio (BER) approaches 0.5 (i.e., total garbage) during the error burst. In other words, an error monitor must be designed to "ride-over" some minimum error burst duration of $t_{b}$ seconds, irrespective of its severity. At the same time, the error monitor should not take too long to pull the link out of service, as this not only subjects messages to long delays, but also results in a large transmit congestion (i.e., a large number of messages in the transmit buffer at the time of link taken-down). Large transmit congestion translates into a large buffer size and long changeover time. Thus, an error monitor should try to keep the transmit congestion as low as possible.

Let $X$ denote the time to take the link out of service, and $M(X)$ some measure of it (e.g., mean, mean $+2 \sigma, 95$ percentile, etc.). A typical behavior of $M(X)$ as a function of BER can be seen in the first chart of Figure 5. Clearly, $M(X)$ should increase as BER decreases. More importantly, for a well-designed algorithm, this curve should show a definite knee. That is, as the BER goes below the knee, $M(X)$ should increase drastically, so that the algorithm will keep the link in service almost indefinitely. On the other hand, as the BER increases above the knee, $M(X)$ should quickly go down to its minimum value dictated by the error-burst ride-over requirement.

An important concept in locating the knee is one of sustainable error rate (SER), i.e., the maximum bit error rate at which the message delay requirements are still satisfied. An error monitor should take the link out of service quickly if the error rate exceeds the SER, and otherwise leave the link in service. That is, the knee should occur at or below SER. It should also be clear that a sharper knee is preferable - ideally, the knee should be "square", i.e., $M(X)=\infty$ for BER $<$ SER, and $M(X)=$ const (determined by the error burst ride-over requirement) if BER $>$ SER. For brevity, a precise characterization of SER is omitted here. For a 5000 mile long link (the longest terrestrial link), the sustainable error rate works out to be around $3.0 \mathrm{e}-5$ for a $64 \mathrm{~Kb} / \mathrm{sec}$ link, $1.0 \mathrm{e}-5$ for a $512 \mathrm{~Kb} / \mathrm{sec}$ link, and $2.0 \mathrm{e}-6$ for a $4 \mathrm{Mb} / \mathrm{sec}$ link. Since these error rates are rather high compared with the typical link quality found in practice; it is okay (and perhaps even desirable) to design the error monitor assuming a lower SER. In this sense, these numbers should be regarded as upper bounds on the error rates at which the link could be left into service for a long time.

(Kant 1995) shows an analytic model of SSCOP performance, which can be used to obtain SER as a function of various network parameters. Clearly, the SER depends on some key link parameters such as link length, link speed, offered load, and message size. This tends to make the error monitor parameters dependent on the link parameters, which is undesirable from an administrative and operations point of view. This issue is 
particularly important in the ATM signalling context because the link parameters may vary over a wide range. In particular, the following ranges must be considered:

- Link Length: 0 to 5000 miles for terrestrial links. Links to geo-synchronous satellite are about 30,000 miles long.

- Link Speed: Currently specified as $64 \mathrm{~Kb} / \mathrm{sec}$ to $4 \mathrm{Mb} / \mathrm{sec}$.

- Offered Load: No filler PDUs $\Rightarrow$ Load may drop to almost zero.

- Message sizes: 1-4 cells with current services. Up To 20 cells in future.

This paper presents an error monitor for in-service SAAL links that meets all the challenges described above. Before inventing this new algorithm, the authors examined the existing error monitoring mechanisms, namely SUERM (signal unit error rate monitor) of low speed $(56 / 64 \mathrm{~Kb} / \mathrm{sec})$ CCS links (BCR 1991), EIM (errored interval monitor) of highspeed $(1.5 \mathrm{Mb} / \mathrm{sec})$ CCS links, and the error monitoring provided by the ATM/SONET layers. None of these were found entirely satisfactory for SSCOP environment.

\section{ALGORITHM DESCRIPTION}

Although the algorithm is not inherently tied to SSCOP, we describe it here specifically for SSCOP. A description of SSCOP may be found in (Kant 1995) and (Quinn 1993). SSCOP assigns a sequence number to all the user PDUs to keep track of retransmitted PDUs and to ensure that the PDUs are delivered in the correct order to the higher layer on the receive side. When the receiver detects a new gap in the received sequence numbers, it alerts the transmitter via a ustat message, which contains a list of missing sequence numbers. In response, the transmitter retransmits those PDUs. In addition, the transmitter periodically sends a poll message, to which the receiver responds via a stat message, which lists all existing gaps in received sequence numbers. The transmitter retransmits these messages. Unnecessary retransmissions are avoided by the protocol using some mechanisms detailed in (Quinn 1993). The poll-stat combination also provides the "I-am-alive" functionality between peers. In particular, there is a timer known as the no-response timer which times the gaps between successive stat arrivals on the transmit side. If this timer expires, the link (or the virtual circuit) is reset.

The proposed error monitoring algorithm resides in the SAAL Layer Management and needs the following pieces of information from SSCOP for its operation:

1. End of a polling interval, i.e., sending of a poll by SSCOP, and the following auxiliary information:

- The current $\mathrm{VT}(\mathrm{S})$ value (i.e., the sequence number to be given to the next user PDU that is to be transmitted for the first time).

- Indication of any retransmissions in the last polling interval (rexmitflag).

- Number of stats received in the last polling interval (n_stats).

2 Indication of a credit rollback by the peer receiver. 
Currently, SSCOP only reports total number of retransmissions during a polling interval, which can be used to extract "rexmit-flag". Other information is, however, available within SSCOP, and can be easily reported to the layer management.

The basic idea behind the error monitoring algorithm is as follows. At the end of each polling interval, a "penalty" factor is computed for the polling interval. The algorithm uses penalty factors over a block of $N_{b l k}$ consecutive polling intervals and computes a quality of service (QoS) measure for the block. This QoS is simply the arithmetic average of the penalty factors, clipped at 1.0. That is, if the average penalty over the block exceeds 1.0, the QoS is made 1.0. Finally, the block QoS is used to compute an overall (or running) QoS by using exponential smoothing over consecutive block QoS's. That is, if Q denotes the running QoS, and $Q_{b}$ denotes the QoS from the current block, $\mathrm{Q}$ is updated as follows:

$Q=(1-\alpha) Q+\alpha Q_{b}$

where $\alpha$ is the exponential smoothing factor in the range $(0,1)$. Whenever the running QoS Q exceeds a threshold thres, the link is taken out of service. The penalty factor over an interval is computed as follows: If no stat has been received for the last $N_{\text {gap }}$ polling intervals, set penalty to $N_{b l k}-1$; otherwise, set it to 1 or 0 depending on whether "rexmit_flag" is set.

At moderate to low error rates, the behavior of this core algorithm is primarily governed by retransmissions of user PDUs. Consequently, the take-down time will increase as the offered user load drops. However, the error monitor should be capable of detecting unacceptable error rates even at zero offered load, since all links may experience long idle periods. Links that are used as alternate routes would almost always be idle. This motivated the addition of the following mechanism to the core algorithm:

The auxiliary mechanism counts the number of stats received (denoted $N_{s r}$ ) over a "super-block" of size $N_{\text {sup }}$ polling intervals. At the end of the super-block, if the number of missing stats (given by $N_{\text {sup }}-N_{s r}$ ) exceeds a threshold $N_{\text {loss }}$, the link is taken out of service. Otherwise, we repeat the process over the next super-block.

This mechanism is almost independent of the load and enforces a minimum link quality depending on the choice of $N_{\text {sup }}$ and $N_{\text {loss }} . *$ It also complements the core monitor at low loads by limiting the failure time.

The algorithm also includes provision to handle situations where the receiver causes retransmissions by withdrawing credit for already transmitted PDUs. This is done by ignoring retransmissions after such a credit rollback until a poll with a higher VT(S) value is sent out.

Although it is convenient to describe the algorithm in terms of counting variables $N_{\text {gap }}, N_{\text {blk }}, N_{\text {sup }}$, and $N_{\text {loss }}$, their use makes the algorithm parameterization heavily dependent on the polling interval $T_{\text {poll }}$. To remove this dependence, it is better to use time-unit versions of these quantities, henceforth denoted by replacing " $\mathrm{N}$ " by " $\mathrm{T}$ " in the notations. The relationship between the two is simply $N_{x}=T_{x} / T_{\text {poll }}$, for $x=g a p$, blk, sup, loss.

The primary parameters of the algorithm and their recommended values are listed in

\footnotetext{
*A slight dependence on load exists because SSCOP resets the keep-alive timer whenever a stat is received during an idle period.
} 
Table 1 Primary parameters of the error monitor and their default values

\begin{tabular}{llclll}
\hline Parm & \multicolumn{1}{c}{ Meaning } & value & Parm & \multicolumn{1}{c}{ Meaning } & value \\
\hline$T_{\text {gap }}$ & Max gap between stats & $0.3 \mathrm{sec}$ & $T_{\text {blk }}$ & Block size & $0.3 \mathrm{sec}$ \\
$T_{\text {sup }}$ & Super-block size & $120 \mathrm{sec}$ & $T_{\text {loss }}$ & Stat loss limit & $1.3 \mathrm{sec}$ \\
$\alpha$ & Smoothing factor & 0.1 & $\beta$ & Clipping threshold & 1.0 \\
thres & Threshold for failure & 0.191 & & & \\
\hline
\end{tabular}

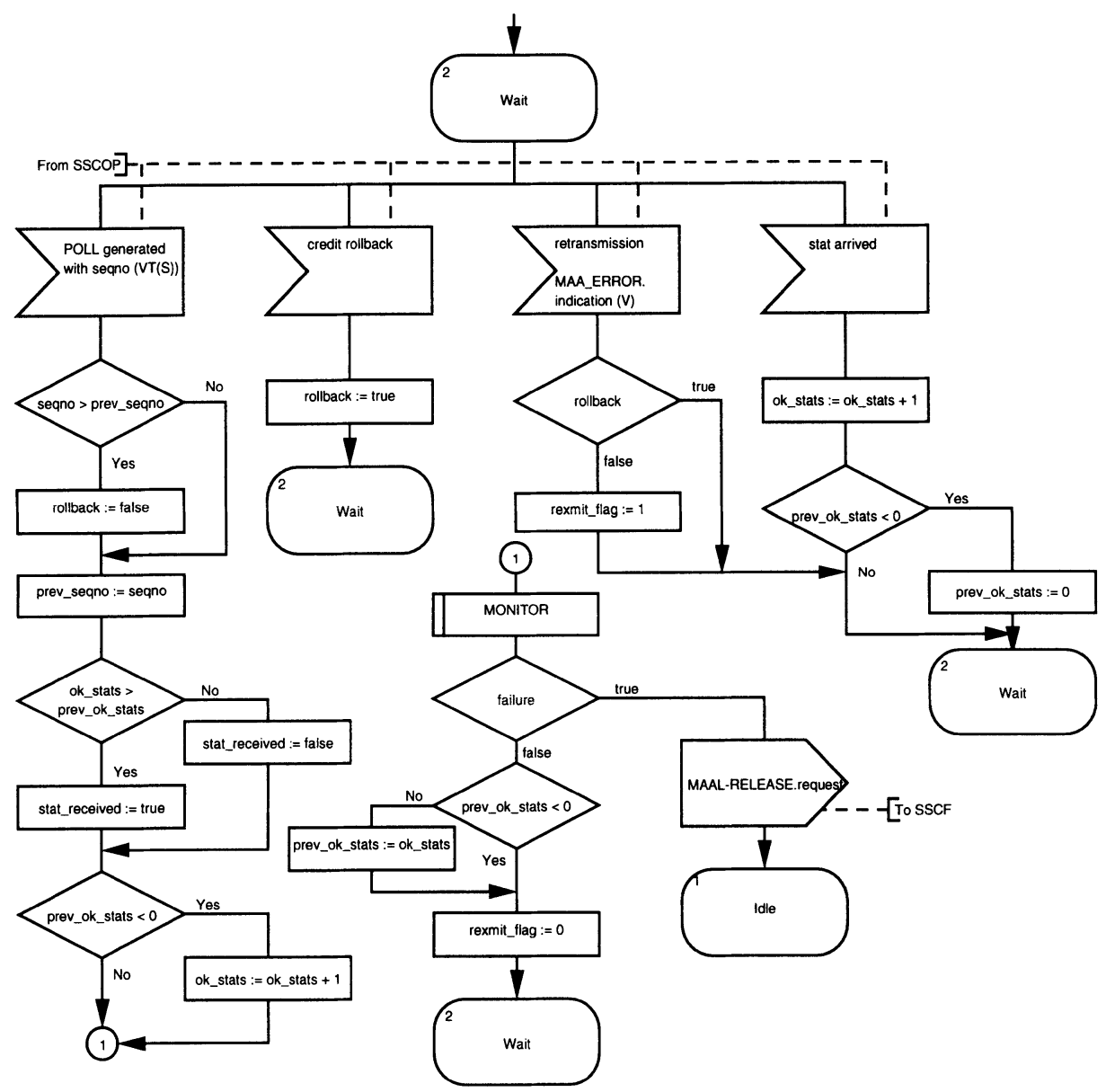

Figure 1 Top Level of the Error Monitor 
Table 1. Figures 1 and 2 show the flow chart of the entire algorithm. The algorithm uses a number of variables, which are described below:

- $N_{\text {gap }}, N_{\text {blk }}, N_{\text {sup }}, N_{\text {loss }}$ : counter versions of $T_{\text {gap }}, T_{\text {blk }}, T_{\text {sup }}, T_{\text {loss }}$.

- PI_count: A counter for polling intervals (PIs).

- ok_stats: A counter for number of received stats in a superblock.

- prev_ok_stats: Remembers ok_stats value for the previous PI.

- stat_log[$\left[N_{\text {gap }}\right]$ : A boolean array to hold stat-arrival indication over last $N_{\text {gap }}$ PI's.

- stat_received: A boolean indicating stat reception in the last polling interval.

- tot_penalty: Running total of penalties over PIs of a block.

- block_qos: QoS measure over a block.

- tot_qos: Overall running QoS measure.

- seqno: $\operatorname{VT}(\mathrm{S})$ at the time of most recent sending of a poll.

- prev_seqno: VT(S) value from the previous polling interval.

- rollback: A boolean to indicate a credit rollback by the peer receiver.

Figure 1 shows the interactions between the error monitor and SSCOP. The conversion of time parameters to counting parameters and auxiliary variable initializations are not shown. Most auxiliary variables are initialized to 0 (or false in case of booleans); the only exceptions being prev_ok_stats (initialized to -1) and stat_log array (initialized to all true). These two initializations are needed to make the algorithm pretend that no PDUs are lost until the arrival of the first stat.

After initialization, the algorithm waits for a trigger from SSCOP and takes appropriate action. Whenever SSCOP reports retransmissions, the error monitor sets rexmit flag unless the rollback flag is set (since that condition indicates that the retransmission may be due to credit rollback.) Whenever SSCOP reports arrival of a stat, ok_stats is incremented. Also, if prev_ok_stats is negative, it is set to zero to indicate the arrival of the first stat. When SSCOP reports a credit rollback, the flag "rollback" is set. When SSCOP reports the expiry of poll timer, seqno, prev_seqno, and rollback are updated. Also, stat_received is set to indicate whether a stat was received in the last polling interval. It can be verified that this boolean will evaluate to true until the first stat is received. Incrementing of ok_stats on each poll reception before the first stat has arrived (i.e., while prev_ok_stats is negative) ensures that stat and poll counters run in sync until the first real stat arrives.

Following these updates, the main body of the error monitor, called MONITOR, is run. In case of a normal exit from the MONITOR (i.e., if a changeover is not declared), prev_ok_stat is updated if it is nonnegative (i.e., only after the very first stat has arrived). The rexmitflag is also reset upon return from MONITOR.

The MONITOR routine, shown in Figure 2, works as follows. The tot-penalty is incremented by $N_{b l k}-1$ if no stat has arrived for the last $N_{g a p}$ intervals. Otherwise, it is incremented by rexmitflag. If the current polling interval ends a block, tot_qos is computed and compared against thres. If the current polling interval ends a superblock, a check is made to see if more than $N_{\text {loss }}$ stats were lost. The flow-chart assumes that the superblock size is an integer multiple of the block size. 

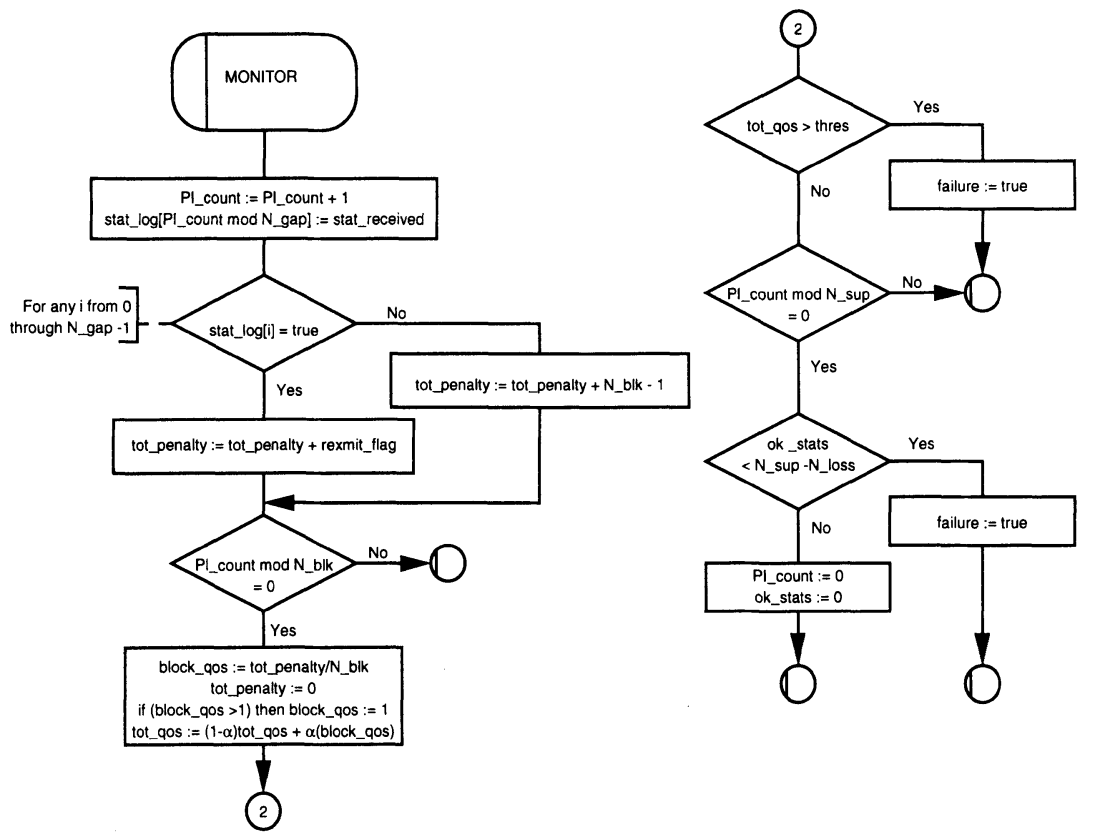

Figure 2 MONITOR routine of Error Monitor

\section{RATIONALE FOR PARAMETER CHOICES}

The most critical parameter for an error monitor is $t_{b}$, the duration of the error burst that must be tolerated. From experience with CCS networks, it is known that a suitable value for $t_{b}$ is $0.3-0.4$ secs. Since riding over a longer burst entails higher transmit congestion, the design here enforces $t_{b}=0.4$ only for bit error ratio (BER) approaching 0.5 . Consequently, the overall algorithm rides over $0.3 \mathrm{sec}$ error burst in nearly all cases, but may not always ride-over $0.4 \mathrm{sec}$ error bursts (although it does so in most cases).

In order to minimize transmit congestion at changeover time, it can be shown that $N_{b l k}$ should be about one-half of $t_{b} / T_{\text {poll }}$. (The factor $1 / 2$ comes from the fact that an error burst can start anywhere within a block, and thus can always span two blocks irrespective of how large the block is.) To minimize link take-down time under severe errors, $T_{\text {gap }}$ should be set as small as possible subject to the constraint that normal jitter in stat reception should not result in nonzero penalty. This leads to the choice of $T_{\text {gap }}=0.3 \mathrm{secs}$ assuming that the SSCOP implementation gives the highest priority to polls/stats/ustats as stipulated in the SSCOP specification (i.e., assuming that the queuing delays suffered by polls and stats are always less than $0.1 \mathrm{sec}$ ). 
The need for clipping and the choice of $\beta=1.0$ is tied to the use of penalty $>1$ in connection with excessive gap between successive stats. The purpose of clipping is simply to limit blk_qos to the same value as the one without the use of penalty $>1$. In this sense, $\beta=1.0$ is the only possible choice. The main reason for not altering the limit on blk_qos is that excessive loss of stats can occur only at very high error rates; at other error rates the algorithm should behave as if the stat-gap mechanism was not present. The choice of $\beta=1.0$ can be justified in other ways too; in fact, a $\beta$ other than 1 is unlikely to be useful; for this reason, the implementation in Figure 2 does not even regard $\beta$ as an algorithm parameter.

The main motivation for monitoring the gap between successive stats comes by considering the algorithm performance for long messages at high error rates. In such cases, the message error probability could be almost 1 whereas the cell error probability is still not too high. In this case, almost no ustats will be generated and the error reporting happens almost entirely via stats. This tends to increase link take-down times substantially in a very narrow range of error rates. By using a nonzero penalty when stats also do not arrive, one can partially compensate for the lack of ustats and thereby bring down this peak in take-down time. Nevertheless, the congestion can still increase substantially in narrow BER range for very long messages.

The rationale for choosing penalty $=N_{b l k}-1$ when the inter-stat gap exceeds $T_{g a p}$ is as follows: It is desirable to set penalty to the highest possible value to minimize link take-down time in the situation described above. Given $\beta=1.0$, the largest useful value of penalty is $N_{b l k}$. This value is fine from an error burst ride-over perspective; however, if stats were lost during the error burst, and the first stat to arrive following the error burst takes more than $200 \mathrm{~ms}$, the link will be taken out of service. Setting penalty $=N_{b l k}-1$ eliminates this problem.

The two remaining parameters of the core algorithm, namely $\alpha$ and thres, can be interrelated by the error burst ride-over requirement. Suppose that a clean link is hit by an error burst of length $t$ seconds with BER approaching 0.5 , after which the link again becomes clean. It is then easy to compute the maximum value of QoS, say $q$, as a function of $\alpha$ for any given scenario of PDU losses due to the error burst. Let this event. Let $q_{\max }$ denote the maximum $q$ over all the scenarios. Then, it is required that $q<$ thres iff $t \leq t_{b}$. Such an analysis indicates that thres $=\alpha(2-\alpha)$. At lower BERs, such a choice does not necessarily ride over error bursts of length $t_{b}$ at all BER values. However, the region of vulnerability is rather small as the results show later.

The design approach is now to choose $\alpha$ and then compute thres from the above equation. With such a design, the maximum transmit congestion does not change substantially as $\alpha$ is decreased. By using an analytic model, it is possible to characterize the behavior the algorithm rather accurately at low error rates (i.e., when the take-down time is at least a few seconds). This model shows that a small $\alpha$ is preferable for two reasons: (a) it results in a sharper knee, and (b) the variability in the take-down time goes down. However, since thres also decreases with $\alpha$, the knee tends to move towards a lower BER. Thus, to ensure that the knee BER for a $4 \mathrm{Mb} / \sec$ link does not become too small, $\alpha$ is chosen as 0.1 .

To parameterize the auxiliary mechanism, it is best to set $T_{\text {loss }}$ to the minimum possible value, and then determine the minimum value of $T_{\text {sup }}$ needed to enforce a desired 
link quality at zero load. Anticipating that $T_{\text {sup }}$ will not be much more than a few minutes, it suffices to allow for stat losses due to one $t_{b}=0.4$ secs error burst and 2 random errors. The error burst can wipe out at most $t_{b} / T_{\text {poll }}+2$ stats. The two random errors can wipe out 2 more stats. Stats may also get delayed due to queuing delays suffered by polls or the stats generated by the polls. We assume that this cumulative delay, say $T_{q d}$, does not exceed 0.5 secs. It follows that $T_{\text {loss }}=t_{b}+4 T_{\text {poll }}+T_{q d}$, which works out to be 1.3 secs.

Given $T_{\text {loss }}$, it is then easy to estimate $T_{\text {sup }}$ to enforce a given link quality, say $\theta$ under random errors. Here $\theta$ is defined as the BER at which the auxiliary mechanism should leave the link in service with a high probability. It is adequate to choose $\theta$ as the sustainable error rate of a $4 \mathrm{Mb} / \mathrm{sec}$ link at normal engineered load. The idea is that an idle in-service link should be good enough to suddenly take normal engineered load without causing excessive delays. From these considerations, $\theta=5.0 \mathrm{e}-6$, which, in turn, gives $T_{\text {sup }} \approx 120$ secs. (A precise setting of $T_{\text {sup }}$ is unnecessary; for implementation ease, it helps to make $T_{\text {sup }}$ a multiple of $T_{b l k}$.)

\section{EVALUATION OF THE ALGORITHM}

The evaluation of the algorithm was done via a detailed simulation of SSCOP. Most results were obtained under "double-sided errors", i.e., when both forward and backward directions experience synchronized error bursts simultaneously. This scenario is more appropriate since it results in worse performance (because the loss of ustats/stats yields less information about the existing error conditions.)

A tolerable burst length of $t_{b}=0.4 \mathrm{sec}$ can wipe out at most 6 stats, which creates a gap of 0.7 secs between successive stats. Allowing another $0.1 \mathrm{sec}$ for jitter, the minimum value of the SSCOP no-response timer $T_{n r}$ is $0.8 \mathrm{secs}$, and was used in all the experiments. At very high error rates, it is the no-response timer that will take the link out of service. The algorithm, however, is not totally dependent on a low setting of no-response timer. In particular, without a no-response timer, the stat-gap mechanism will make the tot_qos exceed the threshold in 3 to 4 blocks (i.e., the link will always be taken out of service in 1.2 secs or less).

The evaluation used three sample link speeds: lowest $(64 \mathrm{~Kb} / \mathrm{sec})$, highest $(4 \mathrm{Mb} / \mathrm{sec})$, and their geometric mean $(512 \mathrm{~Kb} / \mathrm{sec})$. The default link length was $5000 \mathrm{miles}$, although the behavior of the algorithm for other link lengths was also explored. The default (or "normal") message size distribution was $(0.5,0.2,0.1,0.1,0.1)$, i.e., $50 \%$ of the PDUs are 1 cell long, $20 \% 2$ cells long, etc. This distribution is a rough estimate for initial BISUP traffic. Several other distributions were also used in the experiments, a few of which are reported here.

For ease of reference, the offered loads are expressed as percentage of failure-mode engineered load. The latter is chosen as 0.8 Erlang (including polls and stats). With a constant polling interval of $100 \mathrm{~ms}$, the corresponding user load is $0.668,0.783$, and 0.798 Erlangs respectively, for a $64 \mathrm{~Kb} / \mathrm{sec}, 512 \mathrm{~Kb} / \mathrm{sec}$, and $4 \mathrm{Mb} / \mathrm{sec}$ link. These loads are referred to as $100 \%$ loads. The normal engineered load, which is the default, is half as much, or $50 \%$. The major performance parameters for evaluation are as follows: 
Take-down time: Time to declare changeover (i.e., to send a COO message) from the time the error burst starts.

Transmit Congestion: Total number of unacknowledged messages (measured in cells) in the system at the time of changeover order. In order to avoid the question of how full the cells are, congestion was measured in terms of cells rather than PDUs or bytes.

PDU delay: Total delay (time of delivery to the higher layer on receive end minus the time of arrival on the transmit side) experienced by all PDUs that get through before changeover.

Burst ride-over: The probability that the algorithm will fail to ride-over an error burst of a given duration.

For the first 3 parameters, we report the mean and " $m+2 s$ " (mean plus twice the standard deviation). All graphs show two different scenarios; for each scenario, the upper curve is for " $\mathrm{m}+2 \mathrm{~s}$ " value, and the lower one is for the mean value.

$64 \mathrm{~Kb} / \mathrm{s}$, normal msgs, $50 \%$ \& $10 \%$ load

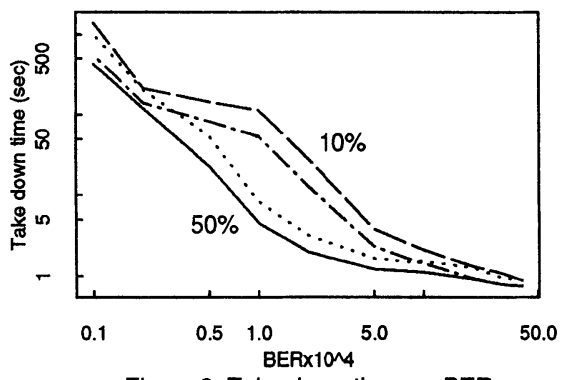

Figure 3: Take-down time vs. BER

$4 \mathrm{Mb} / \mathrm{s}$, normal msgs, $50 \%$ \& $10 \%$ load

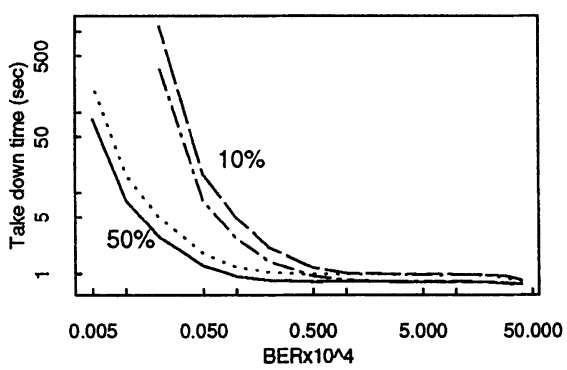

Figure 5: Take-down time vs. BER
$64 \mathrm{~Kb} / \mathrm{s}$, normal msgs, $50 \% \& 10 \%$ load

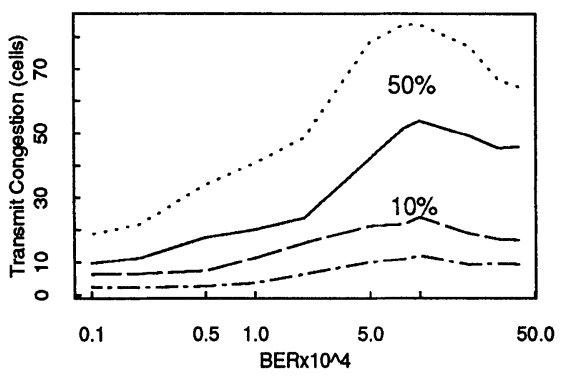

Figure 4: Transmit congestion vs. BER

$4 \mathrm{Mb} / \mathrm{s}$, normal msgs, $50 \% \& 10 \%$ load

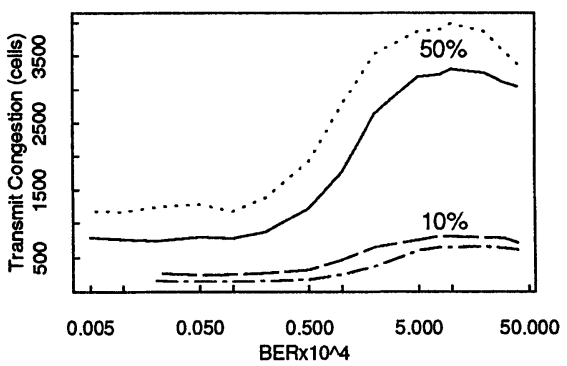

Figure 6: Transmit congestion vs. BER

Due to space limitations, only a few graphs are included here, however, the discussion draws upon a large number of other cases as well. Figures 3 and 5 show the link takedown time as a function of BER for a 5000 mile carrying "normal" messages. These graphs show performance for two different link speeds $(64 \mathrm{~Kb} / \mathrm{sec}$ and $4 \mathrm{Mb} / \mathrm{sec})$ and two different offered loads $(50 \%$ and $10 \%)$. In the low BER region the simulation was 
terminated if the link survived for more than 50 minutes (an arbitrarily chosen cut off point based on the available computing resources). It is seen that the curves have a rather sharp knee that moves appropriately with the link speed. (The $64 \mathrm{~Kb} / \mathrm{sec}$ curve also rises very sharply below a BER of $1.0 \mathrm{e}-5$.) For normal messages at $100 \%$ load, the cutoff BERs were $5.0 \mathrm{e}-6,1.0 \mathrm{e}-6$, and $1.0 \mathrm{e}-7$ respectively for $64 \mathrm{~Kb} / \mathrm{s}, 512 \mathrm{~Kb} / \mathrm{s}$ and $4 \mathrm{Mb} / \mathrm{s}$ links. For $50 \%$ load, these BERs were 5.0e-6, 2.0e-6, and 2.0e-7. These BERs are about 1/10th of the sustainable error rates (SERs) for those links. This is perhaps a bit too conservative, but should be satisfactory in all cases except when a $4 \mathrm{Mb} / \mathrm{sec}$ link is implemented in copper (as opposed to fiber).

As the offered load decreases, the core algorithm has fewer and fewer messages to work with, which tends to increase the take-down time. (See Figure 5). To an extent this is a desirable behavior since the SER increases as the load drops; however, when the load becomes too small, the core algorithm may keep the link in service for a long time at error rates that are generally considered too high. Furthermore, as the offered load approaches zero, the core algorithm will become totally ineffective since there are no retransmissions to monitor. This is where the auxiliary algorithm involving superblocks comes into play. This auxiliary algorithm takes the link out in 2 minutes or less irrespective of the load or link speed up to an error rate of about 2.0e-5. At a BER of $1.0 \mathrm{e}-5$, the algorithm takes about 10 minutes, and at a BER of $5.0 \mathrm{e}-6$, it takes more than 50 minutes. For a 64 $\mathrm{Kb} / \mathrm{sec}$ link, the SER is higher than the $\theta$ for the auxiliary algorithm; therefore, the latter comes into play even at $50 \%$ offered load, as seen by the rather slow ascent of take-down time below a BER of $5.0 \mathrm{e}-5$. At $10 \%$ load, the auxiliary algorithm comes into play at an even higher BER. This explains the somewhat irregular shape of curves in Figure 3. In contrast, at $4 \mathrm{Mb} / \mathrm{sec}$, the SER is much less than $\theta$ even at $10 \%$, and thus one sees only the core algorithm operating in Figure 5.

Figures 4 and 6 show the transmit congestion for the two scenarios discussed above. It is seen that the transmit congestion shows a unimodal behavior, with the maximum typically occurring around 1.0e-3. This error rate is just below the error rate when most of the take-downs will be due to no-response timer. At higher error rates, the congestion trails off because the no-response timer will consistently take the link out of service in about 0.8 seconds with very little variability. If the no-response timer is set to a high value, this effect will not occur and the congestion will continue to increase beyond the BER of 1.0e-3. However, this further increase cannot be too much, since if a stat is not received for more than 2 blocks, the threshold will be crossed at the end of third block. That is, without the no-response timer, the link will be taken out of service in 3 to 4 blocks at very high error rates.

A more careful examination of link take-down times shows that the algorithm adjusts itself automatically in the right direction as the link-speed, message size, or offered load change. Figures 3 and 5 show this effect with respect to link speed and offered load. As the message size increases, the sustainable error rate decreases, which means that the take-down time should decrease at BERs around the knee and lower. Figure 7 shows this effect; although, the difference is almost indiscernible. At very high BERs, long messages increase take-down time somewhat because there are much fewer messages in a block.

As the message size increases, the peak congestion increases, however, the nature of increase depends on the PDU size distribution. The mechanism at work here is the 
$512 \mathrm{~Kb} / \mathrm{s}$, normal \& 24 cell msgs

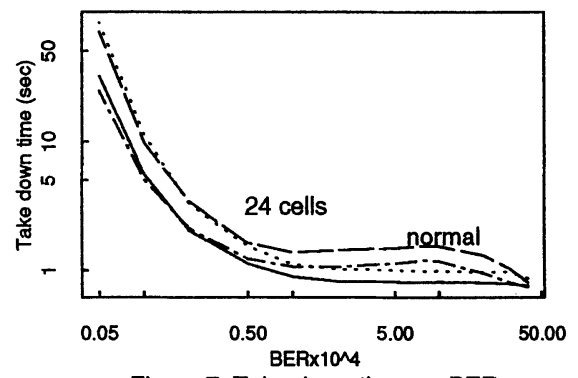

Figure 7: Take-down time vs. BER

$1.5 \mathrm{Mb} / \mathrm{sec}$, normal \& future ISUP msgs

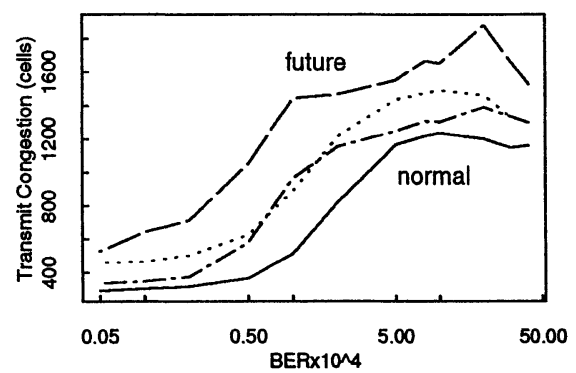

Figure 9: Transmit congestion vs. BER
$512 \mathrm{~Kb} / \mathrm{s}$, normal \& 24 cell msgs

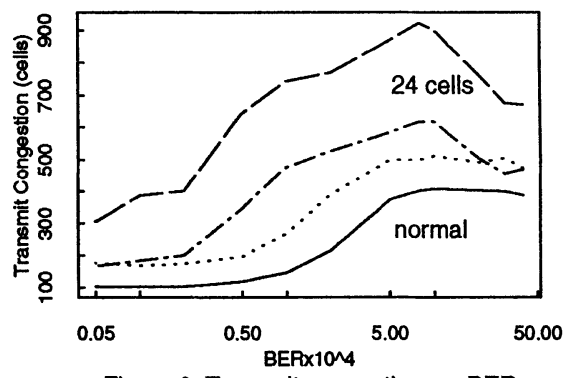

Figure 8: Transmit congestion vs. BER

$512 \mathrm{~Kb} / \mathrm{s}$, normal \& 24 cell msgs

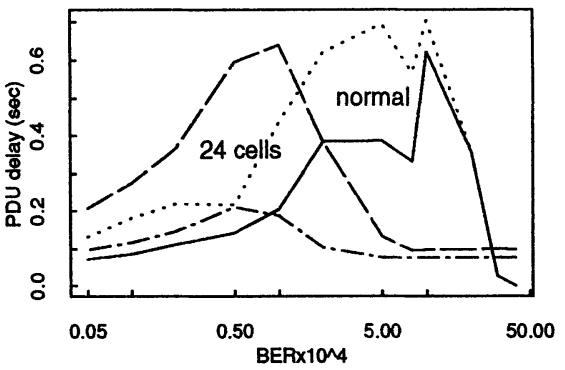

Figure 10: PDU delay vs. BER

lack of ustat generation when a string of long messages is transmitted. Consequently, if most PDUs are very long, very few ustats are generated in the BER range 4.0e-4 and $2.0 \mathrm{e}-3$, which leads to a peak in congestion which may go as high as $25-40 \%$ depending on the message size. The BER region over which the congestion remains high also expands accordingly. This is shown in Figure 8 which compares the transmit congestion for normal and 24 cell messages. However, if a significant percentage of PDUs (say 20-30\%) are short, peak congestion does not increase significantly. For example, the projected BISUP size distribution over 10-20 year window should not be too far from the following distribution that was used for experimenting with a $1.5 \mathrm{Mb} / \mathrm{sec}$ link:

$$
(4,0.75),(8,0.05),(12,0.05),(16,0.05),(20,0.10)
$$

Figure 9 compares transmit congestion for normal messages against the one for this distribution. It is seen that the increase in congestion is no longer very substantial. Therefore, in summary, the algorithm should work with most of the anticipated traffic mixes; however, if the link were to carry very long messages exclusively, it could result in a significant peak in transmit congestion.

Figure 10 shows PDU delays as a function of BER for a $512 \mathrm{~Kb} / \mathrm{sec}$ link for normal and 24 cell PDUs. The PDU delays show a jump at BERs around 5.0e-5 or higher, but remain small overall. It is to be noted that at BERs of $5.0 \mathrm{e}-5$ or more, the link will be 
Table 2 Probability of not riding over a $0.4 \mathrm{sec}$ error burst

\begin{tabular}{crrrr}
\hline BER & short,ss & long,ss & short,ds & long,ds \\
\hline $2.0 \mathrm{e}-4$ & 0 & 0 & 0.005 & 0.01 \\
$5.0 \mathrm{e}-4$ & 0.006 & 0 & 0.015 & 0.037 \\
$8.0 \mathrm{e}-4$ & 0.025 & 0.02 & 0.21 & 0.17 \\
$1.0 \mathrm{e}-3$ & 0.063 & 0.14 & 0.25 & 0.30 \\
$2.0 \mathrm{e}-3$ & 0.25 & 0.19 & 0.37 & 0.29 \\
$4.0 \mathrm{e}-3$ & 0.35 & 0.37 & 0.20 & 0.14 \\
$6.0 \mathrm{e}-3$ & 0.20 & 0.18 & 0.023 & 0.015 \\
$8.0 \mathrm{e}-3$ & 0.13 & 0.089 & 0.005 & 0.006 \\
$1.0 \mathrm{e}-2$ & 0.053 & 0.057 & 0 & 0 \\
$1.4 \mathrm{e}-2$ & 0.005 & 0.005 & 0 & 0 \\
$1.6 \mathrm{e}-2$ & 0 & 0 & 0 & 0 \\
\hline
\end{tabular}

taken out of service in a matter of seconds (see Figure 7); therefore, a large PDU delay in this range is inconsequential.

By analyzing various error burst scenarios, it can be concluded that the burst rideover will be poorest for a heavily loaded high speed link. Consequently, the ride-over tests were performed using a fully-saturated $512 \mathrm{~Kb} / \mathrm{sec}$ and $4 \mathrm{Mb} / \mathrm{sec}$ link. The algorithm was able to ride-over a $300 \mathrm{~ms}$ error burst in all cases. With a $400 \mathrm{~ms}$ error burst, however, there were very narrow regions of BER, where the algorithm did not perform too well. This is to be expected, as stated earlier. Table 2 shows some sample results for $400 \mathrm{~ms}$ ride-over. The four columns after the BER column are for the four combinations of short/long link, and single-sided (ss)/double-sided (ds) errors. A short link is 50 miles, whereas a long link is 5000 miles. Each entry in the table shows the probability that the algorithm will not ride-over a $400 \mathrm{~ms}$ error burst. It is seen that the probabilities become rather large in the range $8.0 \mathrm{e}-4$ to $4.0 \mathrm{e}-3$ and drop off to zero very quickly outside this very narrow range. For example, for BERs above 1.6e-2, the algorithm never fails to ride over the error burst. In practice, the errors can be expected to cover a large BER range, say from 1.0e-3 (or lower) to 0.5 . Assuming a uniform distribution, the probability that the algorithm will fail to ride-over the error burst will be negligible.

In evaluating the algorithm, it is important to examine its sensitivity with respect to various parameters. For example, Figures 11 and 12 compare the take-down time and transmit congestion for a 50 mile (terrestrial) link against a 30,000 mile (satellite) link at $512 \mathrm{~Kb} / \mathrm{sec}$. It is seen that the take-down times are almost identical in the two cases. However, the transmit congestion is much higher and rolls off much more slowly for the satellite link. These characteristics result from the fact that a large number of messages reside in the pipeline for a satellite link. With the same parameterization, the burst tolerance for a satellite link will go down significantly; however, available data indicates that error bursts are typically much shorter for satellite links. Therefore, the same parameterization should work well for satellite links as well.

Figures 13 and 14 compare the performance for a $4 \mathrm{Mb} / \mathrm{sec}$ link when the polling interval is reduced from $100 \mathrm{~ms}$ to $25 \mathrm{~ms}$. (Reduction in polling interval can be beneficial only at very high speeds - see (Kant 1995).) It is easy to show that in this case the 
no-response timer can be decreased to 0.65 secs (from 0.8 secs) without compromising the burst tolerance of the error monitor. Lowering the polling interval has the following two effects on the algorithm performance:

1. The knee of the take-down curve shifts towards higher BER by the same factor as the decrease in the poll timer. Thus, the knee will now occur at a BER of 8.0e-7 instead of $2.0 \mathrm{e}-7$. This is fine (and even desirable) since the sustainable error rate for a $4 \mathrm{Mb} / \mathrm{sec}$ link is $2.0 \mathrm{e}-6$.

2. The congestion remains almost unchanged up to a BER of $1.0 \mathrm{e}-3$, but continues to increase and attains a somewhat higher maximum of 3800 cells at a BER of 3.0e-3. This phenomena can be explained by the fact that 4 times as many stats are being sent, which makes their survival more likely.

$512 \mathrm{~Kb} / \mathrm{s}, 50$ and 30,000 mile length

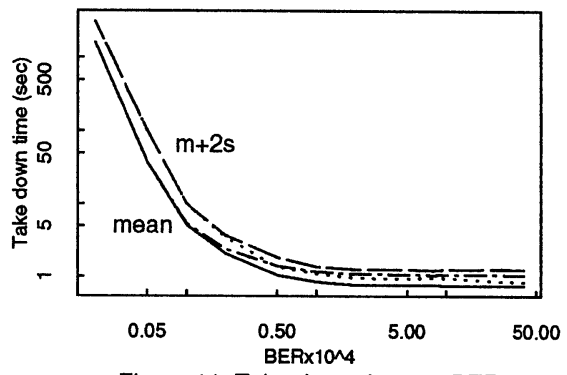

Figure 11: Take-down time vs. BER

$4 \mathrm{Mb} / \mathrm{s}$, poll interval of $100 \& 25 \mathrm{~ms}$

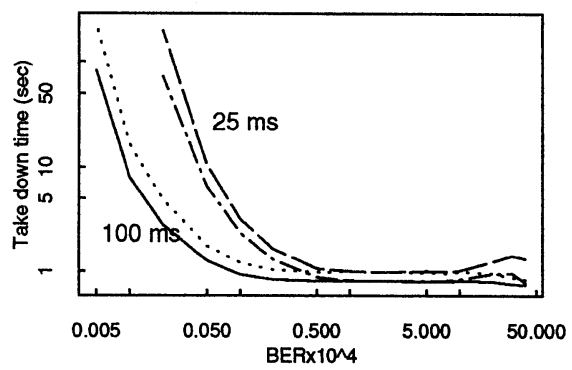

Figure 13: Take-down time vs. BER
$512 \mathrm{~Kb} / \mathrm{s}, 50$ and 30,000 mile length

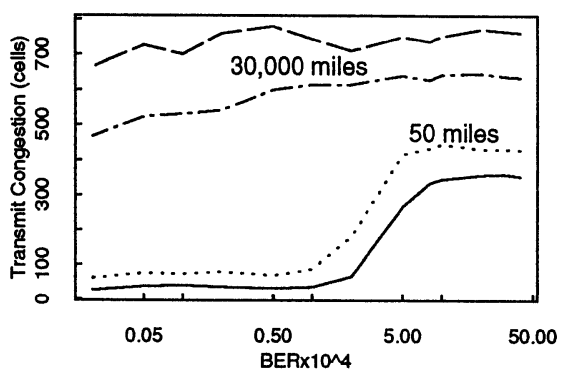

Figure 12: Transmit congestion vs. BER

$4 \mathrm{Mb} / \mathrm{s}$, poll interval of $100 \& 25 \mathrm{~ms}$

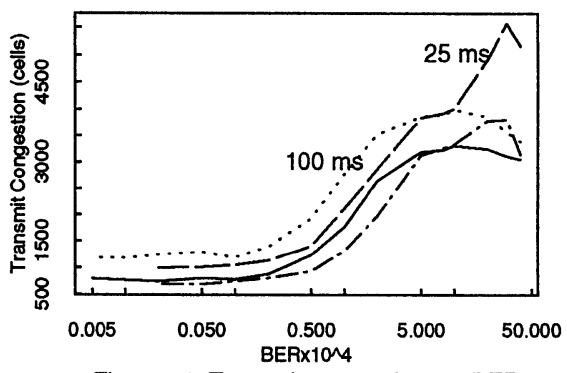

Figure 14: Transmit congestion vs. BER

All experiments until now assumed that the signalling traffic is Poisson. In certain applications (e.g., user to signalling, distance learning, point to multipoint connections, etc.), the offered traffic may be considerably burstier. By using a 2-state MMPP arrival model, it is found that the mean take-down time and mean congestion are not very sensitive to burstiness, but the variance increases noticeably (as expected). 


\section{CONCLUSIONS}

In this paper we presented an algorithm for error monitoring of in-service SAAL links. It was shown that the algorithm adapts itself well over a wide range of link parameters, including link lengths, link speeds, PDU sizes, and offered loads without any changes to the algorithm parameters. Consequently, a single set of error monitor parameters is adequate for almost all links likely to be used in signalling applications. Furthermore, the algorithm works well without any change in parameters even for satellite links. The algorithm also works well without any parameter changes if the polling interval is decreased for high speed links, although this results in a slightly higher peak congestion. Finally, the algorithm is simple, easy to implement, and does not need much interaction with SSCOP. These are important considerations because (1) with high speed links, processors often become a bottleneck, which makes expensive algorithms undesirable, and (2) an algorithm requiring substantial changes to SSCOP standard would be unattractive.

The only negative aspect of the algorithm is a sharp peak in transmit congestion in a narrow BER range when the link carries long messages primarily. Perhaps, the algorithm can be improved further in this area.

\section{REFERENCES}

"Bellcore Specification of the Signalling System Number 7", TR-NWT-246, Issue 2, Vol 2.

Quinn, S. (ed.), "BISDN ATM Adaptation Layer - Service Specific Connection Oriented Protocol", TD PL/11-20C Rev1, 1993.

Kant, K., "Analysis of Delay Performance of ATM Signalling Links", Proc of INFOCOM 95, Boston, MA, April 1995.

Hou, V.T., Kant, K., Ramaswami, V., and Wang, J.L., "Error Monitoring Issues for Common Channel Signalling", IEEE Journal of Selected Areas in Communications, 12, 3, April 1994, pp 456-467.

Ramaswami, V., and Wang, J.L., "Analysis of Link Error Monitoring Protocols in Common Channel Signalling Network", IEEE Trans on Networking, 1, 1, pp 31-47, 1993.

\section{BIOGRAPHY}

Krishna Kant received his Ph.D. degree in Computer Science from The University of Texas at Dallas in 1981. From 1981-1984 he was with Northwestern University, Evanston, IL. From 1985-1991, he was with the Pennsylvania State University, University Park, PA. In 1988 he served with the Teletraffic Theory division at AT\&T Bell Labs, Holmdel, NJ and in 1991 with the Integrated Systems Architecture division at BellCore, Piscataway, NJ. Since Jan 1992, he has been with Network services performance and control group at BellCore, Red Bank, NJ. His research interests include performance modeling of computer and communication systems and fault-tolerant software design. He is the author of a book titled Introduction to Computer System Performance Evaluation (New York: McGrawHill, 1992). 